\title{
Radioactive decay of the late-time light curves of GRB-SNe
}

\author{
Kuntal Misra ${ }^{1 *}$, Andrew Steven Fruchter ${ }^{2}$
}

\author{
${ }^{1}$ Aryabhatta Research Institute of observational sciencES, Manora Peak, Nainital 263002 India \\ ${ }^{2}$ Space Telescope Science Institute, 3700 San Martin Drive, Baltimore MD 21218 USA
}

\begin{abstract}
We present the late-time Hubble Space Telescope observations of two GRB associated supernovae, GRB 030329/SN 2003dh and XRF 060218/SN 2006aj. Using the multi-color data upto $~ 320$ days after the burst, we constrain the late-time decay nature of these supernovae. The decay rates of SN 2003dh are steeper than SN 2006aj. A comparison with two other GRB supernovae, GRB 980425/SN 1998bw and the supernova associated with XRF 020903, shows that the decay rates of SN 2003dh are similar to XRF 020903 and those of SN 2006aj are similar to SN 1998bw. The late-time decay rates are steeper than the ${ }^{56} \mathrm{Co} \rightarrow{ }^{56} \mathrm{Fe}$ radioactive decay rate $\left(0.0098 \mathrm{mag} \mathrm{day}^{-1}\right)$ indicating that there is some leakage of gamma-rays.
\end{abstract}

\section{Introduction}

Gamma Ray Bursts (GRBs) and stripped core collapse supernovae are the spectacular stellar explosions that mark the death of massive stars when their iron core collapses to form a neutron star or black hole releasing enormous amount of energy. The massive progenitors of stripped core collapse SNe (type IIb, Ib, Ic) have lost most of their outer H and He envelope (see Filippenko 1997 for a review). A few supernovae out of the type Ic's show broad spectral lines with very high expansion velocities $(\sim$ $30,000 \mathrm{~km} / \mathrm{s}$ ) and are known as 'broad lined' type Ic supernovae. Till date all supernovae associated with GRBs and X-Ray Flashes (XRFs) which have been spectroscopically typed have been found to be type Ic SNe. Because of the energetic nature of explosion, the supernovae associated to GRBs are often termed as 'hypernovae'. While there is accumulating evidence that nearly all low redshift GRBs have an underlying broad lined type Ic supernova, not all energetic broad lined type Ic's show a GRB association. Till date about a dozen supernovae have been spectroscopically confirmed to have a GRB association (see Cano et al. 2017 for a review).

The supernovae light curves are powered by the radioactive decay of ${ }^{56} \mathrm{Ni} \rightarrow{ }^{56} \mathrm{Co} \rightarrow{ }^{56} \mathrm{Fe}$. The peak bolometric luminosity gives an estimate of ${ }^{56} \mathrm{Ni}$ synthesized in the explosion (Arnett 1982). Typically the broad lined type Ic without a GRB are found to be less luminous with smaller explosion energy and ejecta mass as compared to hypernovae associated to GRBs. The physical parameters for most of the GRB associated supernovae are estimated from the information obtained using the early time light curve and spectrum. It is only in a few cases, like SN 1998bw where late-time data is available, that an estimation of physical parameters is possible (Patat et al. 2001) using the

*kuntal@aries.res.in 
nebular phase data. In the case of GRBs it is not easy to construct late-time light curves of associated supernovae because GRBs are typically distant objects and hence lack detailed study at late-times due to limited data.

In this paper, we present the late-time light curves of two GRB associated supernovae - SN 2003dh/GRB 030329 and SN 2006aj/XRF 060218 which are unique because of the availability of the Hubble Space Telescope (HST) data allowing us to constrain the decay nature of the late-time light curve of the supernovae.

\section{Data reduction}

We retrieved the archival images of GRB 030329/SN 2003dh and XRF 060218/SN 2006aj from the HST archive ${ }^{1}$ which were observed with the Advanced Camera for Surveys (ACS) under the programs GO 9405 (P. I. Fruchter) and GO 10551 (P. I. Kulkarni). HST observed the afterglow of GRB 030329 and the associated supernova SN 2003dh in $F 606 \mathrm{~W}$ and $F 814 \mathrm{~W}$ colors from $\sim 17$ to 772 days after the burst. The last epoch HST observations at $\sim 772$ and 422 days after the burst in $F 606 W$ and $F 814 \mathrm{~W}$ colors respectively are used to estimate the host galaxy flux. At late-times the contribution of the afterglow to the total flux becomes negligible and the supernova flux dominates. We therefore use the HST data beyond $\sim 72$ days after the burst when the contribution from the afterglow becomes insignificant. The late-time observations of XRF 060218/SN 2006aj spanning 150-260 days after the burst were carried out at four epochs in three colors - F555W, F625W, F814W and in three epochs in one color - $F 435 \mathrm{~W}$. We do not have host galaxy images of SN 2006aj free from supernova flux to estimate the host galaxy flux.

The HST data were processed using the multidrizzle routine within the stsdas package in IRAF (Fruchter \& Hook 2002). We used a pixfrac of 0.8 and a scale of 0.03333 to obtain the final drizzled image. The final images have a pixel scale of 0.033 arcsec/pixel. The residual image in GRB 030329 is created by subtracting the last epoch image from the early epochs and the corresponding $\mathrm{AB}$ magnitudes are estimated using the ACS zero points. In the case of SN 2006aj the supernova flux is measured in a small aperture; the total flux thus contains the contribution of the host galaxy within this aperture. The corresponding AB magnitudes are estimated using the ACS zero points.

\section{Analysis}

The unique data set of both SN 2003dh and SN 2006aj allows us to study the late-time behaviour of the light curve. The flux estimates of SN 2003dh are free of any host contamination as discussed in Section 2. To these fluxes we fit an exponential and estimate the late-time decay rates. In the case of SN 2006aj since we do not have host galaxy flux measurements we also fit for the host galaxy flux within the aperture along with the exponetial decline rate. This allows us to simultaneously fit the supernova decay and the underlying host galaxy flux. The decay rates for SN 2003dh and SN 2006aj as obtained from the exponential fits and the corresponding errors are listed in Table 1. We also compare the late-time light curves of SN 2003dh and SN 2006aj to two other GRB associated supernovae GRB 980425/SN 1998bw (Patat et al. 2001) and the supernova associated with XRF 020903 (Bersier et al. 2006). The decay rates of these two supernovae are taken from the literature and are listed in Table 1 for a comparison. A remarkable similarity is seen in the late time decay rates of the GRB associated supernovae. The apparent magnitudes published in the literature are converted

\footnotetext{
${ }^{1}$ http://archive.stsci.edu/hst/search.php
} 


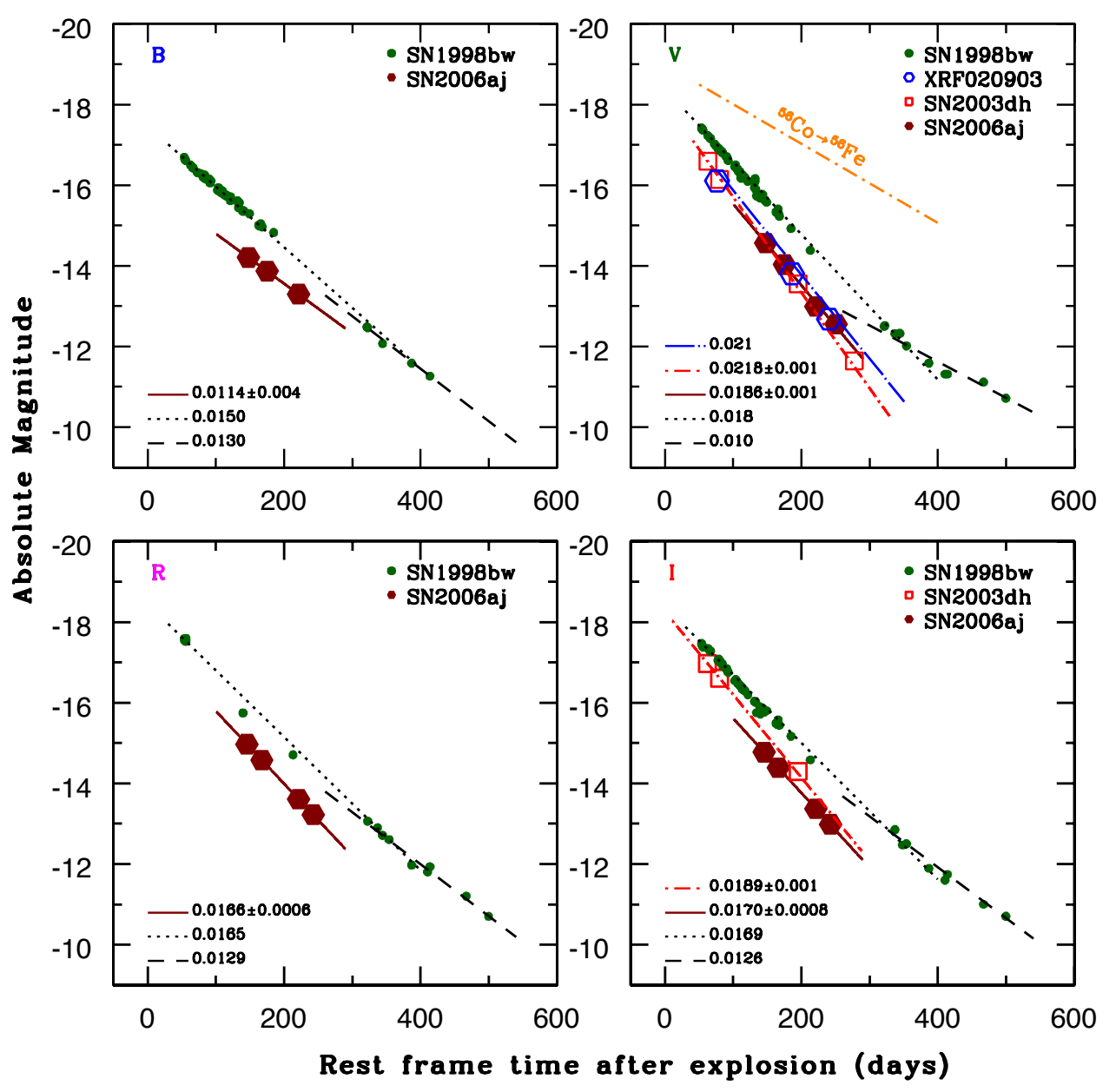

Figure 1: A comparison of multi-color absolute magnitudes of SN 1998bw (Galama et al. 1998; McKenzie et al. 1999; Sollerman et al. 2000; Patat et al. 2001), the supernova associated with XRF 020903 (Bersier et al. 2006), SN 2003dh (This work) and SN 2006aj (This work).

to $\mathrm{AB}$ magnitudes for a direct comparison with $\mathrm{AB}$ magnitudes derived from the HST observations. The absolute magnitudes have been derived from the observed photometry using a cosmology of $H_{0}=70 \mathrm{~km} \mathrm{~s}^{-1} \mathrm{Mpc}^{-1}, \Omega_{m}=0.27$ and $\Omega_{\Lambda}=0.73$. For each supernovae we plot the absolute magnitudes in Fig. 1 corrected for the luminosity distance and total (Galactic+Host) extinction. We see that the decay rates of SN 2006aj are remarkably similar to those of SN 1998bw at late-times except for the $B$ band. On the other hand we see that the decay rates are steeper in SN 2003dh and they are similar to the decay seen in the supernova associated to XRF 020903 (Table 1). In Fig. 1 we have also shown the ${ }^{56} \mathrm{Co} \rightarrow{ }^{56} \mathrm{Fe}$ radioactive decay $\left(0.0098 \mathrm{mag} \mathrm{day}^{-1}\right)$ in the case of complete $\gamma$-ray trapping. We see that the decay rates of all the supernovae shown in Fig. 1 are steeper than the ${ }^{56} \mathrm{Co} \rightarrow{ }^{56} \mathrm{Fe}$ decay rates indicating that there is some leakage of $\gamma$-rays. For a comparison, in SN 1998bw we also show the data in the time range of 324-504 days after the burst (Fig. 1). It is at late phases $(t>300$ days), the multi-band light curves of SN 1998bw show a clear flattening and the light curve settles onto a ${ }^{56} \mathrm{Co} \rightarrow{ }^{56} \mathrm{Fe}$ decay phase. 
Table 1: Late-time decay rates of GRB associated supernovae

\begin{tabular}{cccccc}
\hline \hline Object & $B$ & $V$ & $R$ & $I$ & Reference \\
\hline GRB 030329/SN 2003dh & - & $0.0218 \pm 0.001$ & - & $0.0189 \pm 0.001$ & This work \\
XRF 060218/SN 2006aj & $0.0114 \pm 0.004$ & $0.0186 \pm 0.001$ & $0.0166 \pm 0.0006$ & $0.0170 \pm 0.0008$ & This work \\
\hline $\begin{array}{l}\text { GRB 980425/SN 1998bw } \\
\quad \text { (54-312 days) }\end{array}$ & 0.0150 & $0.018 \pm 0.001$ & 0.0165 & 0.0169 & Patat et al. 2001 \\
$\begin{array}{c}\text { GRB 980425/SN 1998bw } \\
(321-500 \text { days) }\end{array}$ & 0.0130 & 0.010 & 0.0129 & 0.0126 & Patat et al. 2001 \\
$\quad$ XRF 020903/SN & - & $0.021 \pm 0.001$ & - & - & Bersier et al. 2006 \\
\hline
\end{tabular}

\section{PRELIMINARY RESULTS}

We present the late-time HST ACS multi-color observations of two GRB associated supernovae GRB 030329/SN 2003dh and XRF 060218/SN 2006aj. Both these GRB associated supernovae are unique because of the availability of late-time data which allows us to constrain the decay behaviour at late-times. The SN associated with GRB 980425/SN 1998bw and XRF 020903 are others for which late-time observations exist. The decay rates of SN 2003dh and SN 2006aj are remarkably similar at late-times and compare very well with those of SN 1998bw and XRF 020903. We see that the decay rates of GRB associated supernovae are steeper than the ${ }^{56} \mathrm{Co} \rightarrow{ }^{56} \mathrm{Fe}$ decay rate indicating that there is some leakage of $\gamma$-rays.

\section{Acknowledgements}

We thank the organizers of "The First BINA Workshop" in ARIES, Nainital, India to provide an opportunity to present our work.

\section{References}

Arnett W. D. 1982, ApJ, 253, 785

Bersier D., Fruchter A. S., Strolger L.-G. et al. 2006, ApJ, 643, 284

Cano Z., Wang S.-Q, Dai Z.-G., Wu X.-F. 2017, Advances in Astronomy, 2017, id8929054

Filippenko A. V. 1997, ARA\&A, 35, 309

Fruchter A. S., Hook R. N. 2002, Publications of the Astronomical Society of the Pacific, 114, 144

Galama T. J., Vreeswijk P. M., van Paradijs J. et al. 1998, Nature, 395, 670

Iwamoto K., Nakamura T., Nomoto K. et al. 2000, ApJ, 534, 660

McKenzie E. H., Schaefer B. E. 1999, PASP, 111, 964

Patat F., Cappellaro E., Danziger J. et al. 2001, ApJ, 555, 900

Sollerman J., Kozma C., Fransson C. et al. 2000, ApJ, 537, L127 